CORRIGENDUM

\title{
Evolution and genome architecture in fungal plant pathogens
}

Mareike Möller \& Eva H. Stukenbrock

Nature Reviews Microbiology 15, 756-771 (2017)

In Table 1 of this article, the species-specific hosts for both Leptosphaeria species should be crucifers (it was originally written as conifers). The mistakes have been corrected in the PDF and html versions online. The authors apologize to the readers for any confusion caused. 